\title{
Effect of Various Approximations on Predicted Progressive Failure in Plain Weave Composites
}

\author{
John Whitcomb \\ Kanthikannan Srirengan \\ Aerospace Engineering Department \\ Texas A\&M University \\ College Station, Texas
}

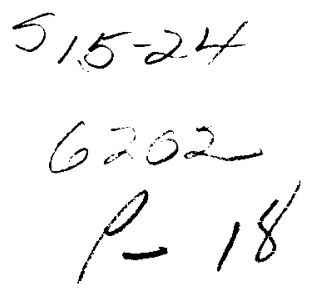

\begin{abstract}
Three-dimensional finite element analysis was used to simulate progressive failure of a plain weave composite subjected to in-plane extension. The loading was parallel to one of the tow directions. The effects of various characteristics of the finite element model on predicted behaviour were examined. The predicted behavior was found to be sensitive to quadrature order, mesh refinement and the material degradation model. Also the sensitivity of the predictions to the tow waviness was studied. The predicted strength decreased considerably with increased waviness. More numerical studies and comparisions with experimental data are needed to establish reliable guidelines for accurate progressive failure prediction.

\section{Introduction}

Textile composites consist of interlaced tows (fiber bundles) which are then impregnated with a matrix material and cured. Figure 1 illustrates the architecture for a plain weave composite. The interlacing of the tows offers the potential for increased through-thickness strength. There is also the potential for reduced fabrication costs, since fairly complicated shapes can be formed using textile machinery. One disadvantage of textiles is the difficulty in predicting their performance. The complex geometry makes detailed stress analysis quite challenging. The early analyses were based on modified laminate theory. ( eg. References 1,2) In recent years there have been a few attempts to discretely model the fiber bundle architecture and predict internal stress states (eg. References 3-11) Reference 11 presented a particularly interesting progressive failure analysis of a plain weave composite. The results in Reference 11 consisted of nominal stress strain curves. The response of the composite was almost linear for in-plane extension and highly nonlinear for in-plane shear. The nonlinearity was primarily a result of progressive damage. However, little information was provided on damage evolution and load redistribution within the composite during the loading process. Also, there was no indication of the sensitivity of the predictions to
\end{abstract}


mesh refinement or other approximations inherent in such analyses.

This paper has two objectives. The first is to evaluate the sensitivity of predicted progressive failure to quadrature order, mesh refinement, and choice of material degradation model. The second objective is to describe the nature of the progressive failure process for two weaves with very different waviness. Loading consisted of a nominally uniaxial stress along one of the fiber tow directions. Only mechanical loads were considered in this study. To simplify the response the composite was assumed to consist of an infinite number of identical unit cells in all three coordinate directions.

The following sections begin with a description of the basic theory used for progressive damage modelling. Then the configurations will be described. Finally the results from the numerical simulations will be discussed.

\section{Theory}

There is no "right" way to model damage evolution in a textile composite that is also practical. It is not feasible to discretely model all of the damage, so approximation is unavoidable. Perhaps the simplest procedure to account for damage in a finite element model is to modify the constitutive matrix at the quadrature points of a numerically integrated finite element. If history effects are not included, the analysis of the loading becomes a series of elastic analyses. Of course, there are many possibilities for how to modify the constitutive matrix. Three techniques were used herein. The first method considered the material totally failed (ie. the entire constitutive matrix was reduced to essentially zero) when any allowable stress component was exceeded. This method will be refered to as the non-selective discount method. The second technique selectively reduced the rows and columns of the constitutive matrix according to the particular stress allowable which was exceeded. For example, if the third stress component exceeded the allowable, the third row was set to essentially zero to eliminate that stress component. To keep the constitutive matrix symmetric, the third column would also be set to zero. Zeroing the column has the undesirable side effect of stiffening the material with respect to the other stresses. This degradation method will be referred to as the selective RC method. The third technique selectively reduced the engineering moduli according to the particular stress allowable which was exceeded. Except as noted, this technique was used in the analyses. This technique will be referred to as the Blackketter method.

The progressive failure analysis begins with a linear analysis of the undamaged configuration. Based on the calculated stresses, the initial load was scaled back so that failure would occur only at points which were within two percent of the maximum normalized stress. (The stresses were normalized by the respective strengths.) The constitutive matrix was modified at the failure points. Residual forces were calculated and used to determine the incremental displacements required to restore equilibrium. The total displacements were updated and used to determine the new stresses. If no further failures occured at the current nominal strain state, the nominal strain was incremented to cause failure. This procedure was repeated until the nominal strain exceeded one percent.

\section{Configurations}

The fiber bundles or tows in the models were generated by translating a lenticular crosssection along a sinusoidal path. The waviness ratio is defined to be the ratio of the woven mat thickness to the wavelength. The weave consists of warp and fill tows oriented perpendicular to each other. In general, the warp and fill tows could be different in terms of material and shape. In 
this study they were assumed to be identical. More details about the mesh geometry can be found in Reference 9. The following subsections describe the finite element meshes, the boundary conditions, and the material properties.

\section{Finite Element Meshes}

Symmetry in the material and loading was exploited so that only $1 / 32$ of a unit cell had to be modeled. A wide range of mesh refinements were used, as shown in Figure 2. The crude mesh had only 4 elements and 42 nodes. The most refined mesh had 192 elements and 1049 nodes. The elements were 20 -node hexahedral elements. Since only $1 / 32$ of the unit cell was modeled, these refinements correspond to full cell models with 128 to 6144 elements.

\section{Boundary Conditions}

The periodic boundary conditions for a complete unit cell are quite simple. The appropriate boundary conditions for a 1/32 unit cell are a bit more complicated. Derivation of the periodic boundary conditions is somewhat tedious, so details will not be given here. Details can be found in Reference 9. The periodic conditions are listed below. Figure 2 shows the coordinate system assumed.

$\begin{array}{llll}u(a / 2, y, z)=u_{0} & v(x, a / 2, z)=\text { constant } & w(x, y, c / 2)=\text { constant } \\ u(0, y, z)=-u(0, y,-z) & v(0, y, z)=v(0, y,-z) & w(0, y, z)=-w(0, y,-z) \\ u(x, 0, z)=u(x, 0,-z) & v(x, 0, z)=-v(x, 0,-z) & w(0, y, z)=-w(0, y,-z)\end{array}$

The load was controlled by specifying the magnitude of $u_{0}$. This corresponds to uniaxial loading in the warp tow direction.

\section{Material Properties}

The unit cell contains two "types" of materials: the tows and the matrix pockets. Relative to the material coordinate system, the properties of the tows are invariant (before damage occurs). Of course, the properties of the tows are needed in the global coordinate system. Fourth order tensor transformation formulas were used to perform the required calculations. The rotation angles to be used in these formulas were specified at each quadrature point by using interpolation. This procedure was shown in References 8 and 9 to be preferable to using a single angle for the entire element. The particular properties used are listed below. These properties, which are 
representative of AS4/3501-6 graphite/epoxy, are from Reference 13.

\begin{tabular}{lrrrrr} 
& \multicolumn{2}{c}{ Tow properties } & \multicolumn{2}{c}{ Matrix properties } \\
\multicolumn{3}{c}{ Modulus } & \multicolumn{1}{c}{ Strength } & Modulus & Strength \\
$\mathrm{E}_{11}$ & $154.27 \mathrm{GPa}$ & $2342.0 \mathrm{MPa}$ & $3.45 \mathrm{GPa}$ & $84.85 \mathrm{MPa}$ \\
$\mathrm{E}_{22}$ & $10.80 \mathrm{GPa}$ & $56.6 \mathrm{MPa}$ & $3.45 \mathrm{GPa}$ & $84.85 \mathrm{MPa}$ \\
$\mathrm{E}_{33}$ & $10.80 \mathrm{GPa}$ & $56.6 \mathrm{MPa}$ & $3.45 \mathrm{GPa}$ & $84.85 \mathrm{MPa}$ \\
$\mathrm{G}_{12}$ & $7.47 \mathrm{GPa}$ & $48.7 \mathrm{MPa}$ & $1.28 \mathrm{GPa}$ & $101.00 \mathrm{MPa}$ \\
$\mathrm{G}_{13}$ & $7.47 \mathrm{GPa}$ & $48.7 \mathrm{MPa}$ & $1.28 \mathrm{GPa}$ & $101.00 \mathrm{MPa}$ \\
$\mathrm{G}_{23}$ & $3.33 \mathrm{GPa}$ & $48.7 \mathrm{MPa}$ & $1.28 \mathrm{GPa}$ & $101.00 \mathrm{MPa}$ \\
$v_{12}$ & 0.28 & & & 0.35 & \\
$v_{13}$ & 0.28 & & 0.35 & \\
$v_{23}$ & 0.34 & & 0.35 &
\end{tabular}

\section{Results and Discussions}

Most of the results in this paper illustrate the effects of characteristics of the finite element model on the progressive failure prediction. The effects of quadrature order, mesh refinement, and material degradation strategy will be considered first. Then the effect of the tow waviness on failure behavior be discussed. Except where indicated otherwise, the results will be presented for a waviness ratio of $1 / 3$ and for the material degradation strategy in Reference 11, ( the "Blackketter method").

Figure 3 shows the effect of quadrature order on the stress-strain curve. The peak stress obtained using 8 quadrature points $(2 \times 2 \times 2)$ is 4 percent higher than that obtained using 64 points. The peak stresses obtained using 27 and 64 quadrature points differ by 1 percent. Damage initiation is predicted 3 percent earlier when 64 points are used. After the large stiffness loss which occurs at about 0.3 percent strain, there is even larger differences in the predictions. In Reference 12, non-selective discount was used for the same configuration. The difference in the peak stress obtained using 8 and 64 point quadratures was 10 percent. Hence, the sensitivity of the predictions to quadrature depends on the degradation model. The sensitivity to quadrature order is not suprising. For example, when more quadrature points are used, the more extensive sampling is more likely to find the extremes in the stress field. One might expect a refined mesh to exhibit less sensitivity to quadrature order than a coarse mesh. For the meshes considered in this study, this was the case. Also, when failure occurs within an element, and the constitutive matrix is modified, the element becomes inhomogeneous. The numerical integration effectively fits a polynomial function to the variation of the material properties. Since the material properties are very different in the failed and unfailed parts of the element, it is difficult to obtain a good fit. In fact, there is a concern as to whether the assumed quadratic displacement functions for a 20node element are sufficient to obtain a reasonable approximation regardless of the integration order.

Figure 4 shows the effect of mesh refinement on the predicted stress-strain curve for the two waviness ratios. The 4 element model predicts the correct trends, but is quite inaccurate. The error in the peak stress is much worse for larger waviness ratio. The peak stress and corresponding strain for a coarse mesh is larger than for a refined mesh, but the peak stress and corresponding strain for a moderately refined mesh are not necessarily bound by the extreme cases of mesh refinement. (see Figure $4 \mathrm{~b}$ ) The peak stress and the corresponding strain, decrease with increased waviness. The ratio of the initial damage stress to the peak stress and the corresponding ratio of strains increase with decrease in waviness ratio. That is, not only is the strength reduced by the 
waviness, but there is also earlier damage initiation relative to the peak stress.

Figure 5 shows damage volume versus the nominal strain for the warp and fill tows for four mesh refinements. Damage volume for the resin is not shown since there is almost no damage. The curves are quite close for the 108 and 192 element meshes, which suggests that the curves might be close to convergence. As the mesh is refined, the increments in damage volume become smaller, but more numerous. The damage volume at the peak stress decreases with mesh refinement for both the warp and fill tows. This is not surprising since the corresponding stress is also smaller. After the peak stress is reached, there is a large increase in damage volume without an increase in strain (of course, the stress drops). The damage volume after this sudden increase is sensitive to mesh refinement for the warp tow but insensitive to mesh refinement for the fill tow. The curve for the 192 element mesh in Figure 5a has points labeled A and B. From A to B the damage initiation and growth was dominated by the inter-tow normal stress $\sigma_{33}$. The sudden increase in damage at point $B$ was due to the stress component $\sigma_{13}$ (the $x_{1}$ direction is parallel to the fibers),

The damage volumes just after the peak stress is reached is as follows

Waviness Ratio Warp Fill Percent Stiffness Loss

$\begin{array}{llll}1 / 6 & .51 & .39 & 41 \\ 1 / 3 & .33 & .35 & 60\end{array}$

Interestingly, the damage volume is larger for the $1 / 6$ waviness ratio, but the percentage stiffness loss is less.

Figure 6 and 7 show the effect of mesh refinement and waviness ratio on damage accumulation during loading. The black region indicates the damage zone. The stress-strain curve for a particular mesh is shown above that mesh. The points labeled A,B and C indicate the correspondence between the strain level and the damage contours. The damage zone corresponding to point $\mathrm{A}$ indicates the initial damage. The damage zones corresponding to points $B$ and $C$ indicate the pre- and post-collapse damage states, where collapse refers to the large stress drop just after the peak stress is reached. Also indicated are the stress components which contributed to the damage. The 4-element mesh does not model the initial failure well for the two waviness ratios of $1 / 3$ and $1 / 6$. The 32 element model performs reasonably well for obtaining qualitative results. Further numerical studies are needed to determine how close the 192 element results are to convergence. For the $1 / 3$ waviness ratio $\sigma_{33}$ dominates the initial failure. This initial failure appears to be an inter-tow failure resembling delamination in laminated composites. The collapse is characterized by $\sigma_{13}$ failure in the warp tows. For the $1 / 6$ waviness ratio, the collapse is due to a significant failure of the warp tow due to $\sigma_{13}$ and cracking of the fill tow due to $\sigma_{22}$.

Figure 8 shows the stress-strain curves obtained using the three degradation models described earlier: non-selective, selective RC, and the Blackketter method. The non-selective method predicts $22 \%$ lower peak stress than the Blackketter method. Selective reduction of rows and columns in the constitutive matrix results in a much larger residual stiffness after the peak stress than the other two methods. Selective reduction of rows and columns in the constitutive matrix also does not result in a large sudden drop in the stress after the peak stress is reached. Concomitantly, for the selective RC method there is also no sudden increase in the damage volume after the peak stress is reached. (This is not shown on the plot.) It should be noted that if variability in the strength of the constituents from one unit cell to another was included, there 
would also be less severe jogs in the stress-strain curve.

\section{Concluding Remarks}

Simulation of progressive failure in a plain weave composite is extremely complex. Consequently, only approximate treatment is practical at this time. One of the goals of this paper was to examine the effect of several approximations on predicted behaviour. One obvious conclusion from this study is that the predictions are quite sensitive to a number of decisions which must be made when assembling a finite element model. Further numerical experiments and comparisons with experimental data are needed to establish guidelines for accurate analysis of progressive failure.

Another objective of this paper was to describe the effect of tow waviness on damage accumulation. The results suggest that the degree of waviness not only affects the stress at which damage initiates, but also the type of damage which occurs. Also, the stress component responsible for damage changed during the progressive failure process.

\section{Acknowledgements}

This research was supported by NASA Grant NAG-1-1324. James Reeder was the technical monitor. This support is gratefully acknowledged.

\section{References}

1. Ishikawa, T. and Chou.T.W.: "Stiffness and Strength Behavior of Woven Fabric Composites," Journal of Material Science, 17:3211-3220, 1982.

2. Ishikawa.T. and Chou T.W.: "Elastic Behavior of Woven Hybrid Composites," Journal of Composite Materials, 16:2-19, January 1982.

3. Paumelle, P., A. Hassim, and F. LÁnÁ.: "Composites with Woven Reinforcements: Calculation and Parametric Analysis of the Properties of the Homogeneous Equivalent," La Recherche AÁrospatiale, 1:1-12, 1990.

\section{Paumelle, P., A. Hassim, and F. LÁnÁ.: "Microstress Analysis in Woven Composite} Structures," La Recherche AÁrospatiale, 6:47-62., 1991.

5. Whitcomb, J. D.: "Three-Dimensional Stress Analysis of Plain Weave Composites," Composite Materials: Fatigue and Fracture, ASTM STP 1110, T. K. O'Brien, Ed., Philadelphia: American Society for Testing and Materials, 3:417-438., 1991.

6. Woo, K.: "Stress and Failure Analysis of Textile Composites," Ph.D. Dissertation, Department of Aerospace Engineering, Texas A\&M University, 1993.

7. Dasgupta, A. and Bhandarkar, S.: "Effective Thermomechanical Behavior of Plain-Weave Fabric-Reinforced Composites Using Homogenization Theory," Journal of Engineering Materials and Technology, $116: 99-105$, January 1994.

8. Avery, William B. and Carl T. Herakovich. 1987. "A Study of the Mechanical Behavior of a 2D Carbon-Carbon Composite," Virginia Polytechnic Institute and State University, Interim 
Report 66.

9. Chapman, C.: "Effects of Assumed Tow architecture on the Predicted Moduli and Stresses in Woven Composite," MS thesis, Department of Aerospace Engineering, Texas A\&M University, 1993.

10. Guedes J.M. and Kikuchi N.,"Preprocessing and Postprocessing for Materials Based on the Homogenization Method with AdaptiveFinite Element Methods," Computer Methods in Applied Mechanics and Engineering, 83 :143-198, 1990.

11. Blackketter, D.; Walrath, D.; and Hansen, A.: "Modeling Damage in a Plain Weave FabricReinforced Composite Material," Journal of Composites Technology and Research, $15: 2$ : 136142, Summer 1993.

12. Whitcomb, J.D. and Srirengan, K.: Simulation of Progressive Failure in Plain Weave Composites. Proceedings of the Symposium on Mechanics of Textile Composites at the ASME International ME'94 Congress \& Exposition, November, 1994.

13. Naik, R.: "Micromechanical Combined Stress Analysis-MICSTRAN, A User Manual NASA Contractor Report 189694, Oct. 1992. 


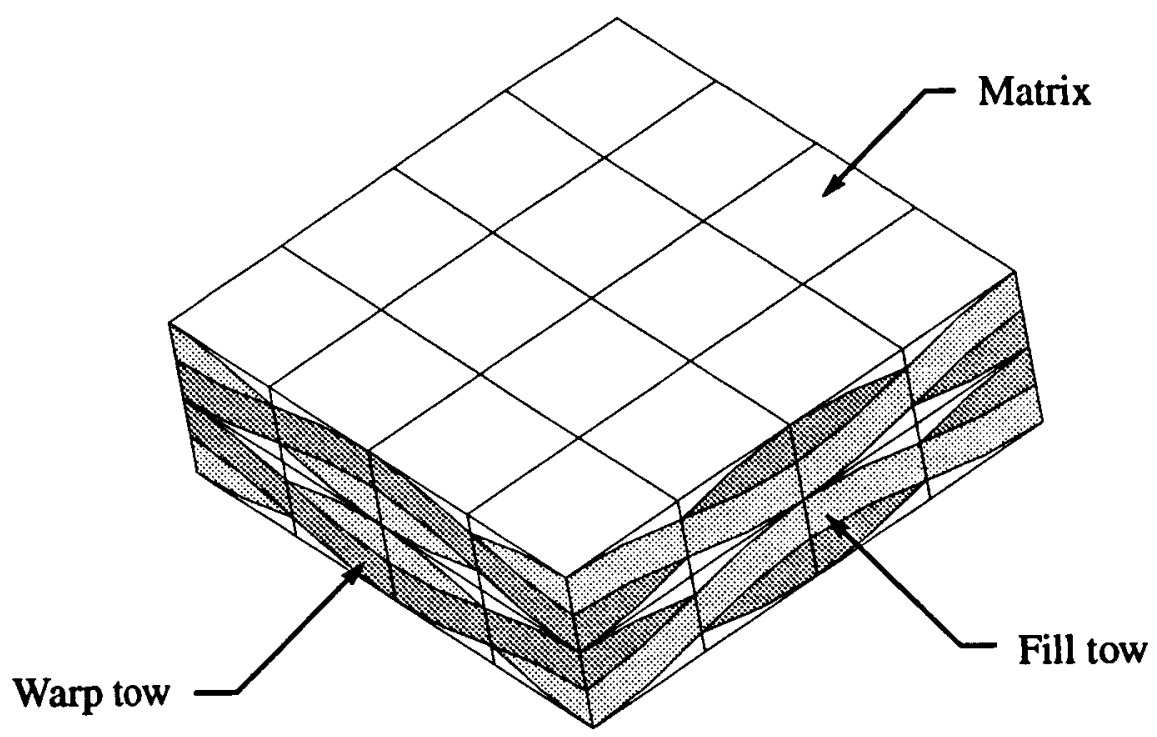

(a) Full unit cell.

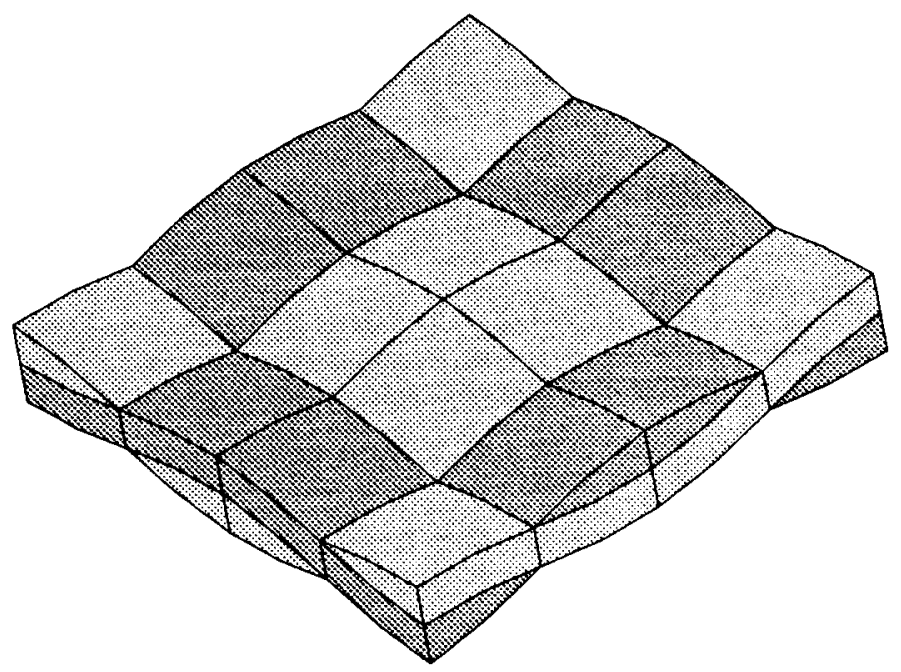

(b) Single mat with matrix pockets removed.

Figure 1 Schematics of plain weave composite. 


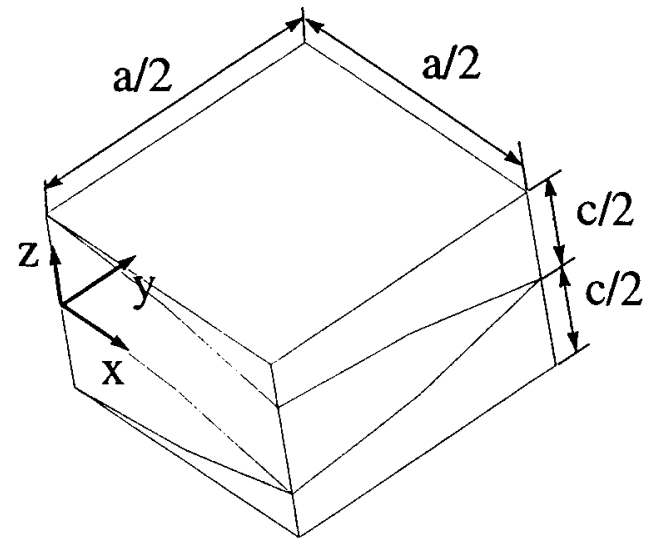

Mesh 1 :

4 elements and 42 nodes

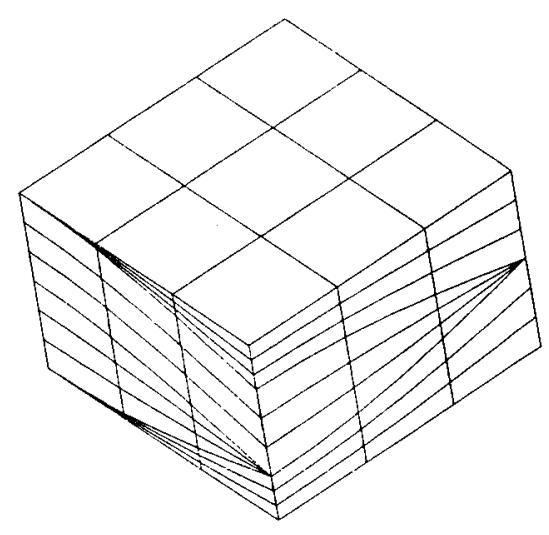

Mesh 3 :

108 elements and 634 nodes

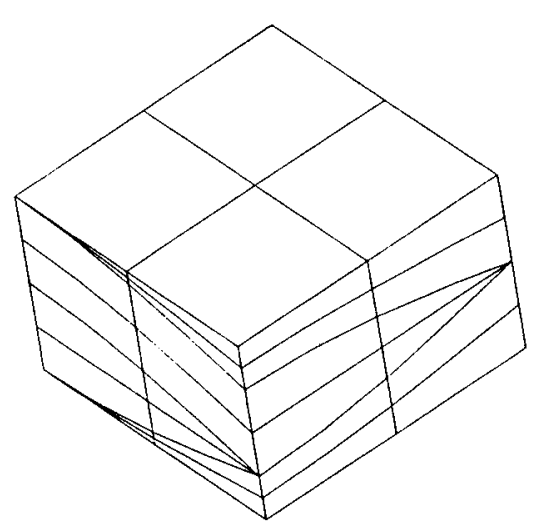

Mesh 2:

32 elements and 221 nodes

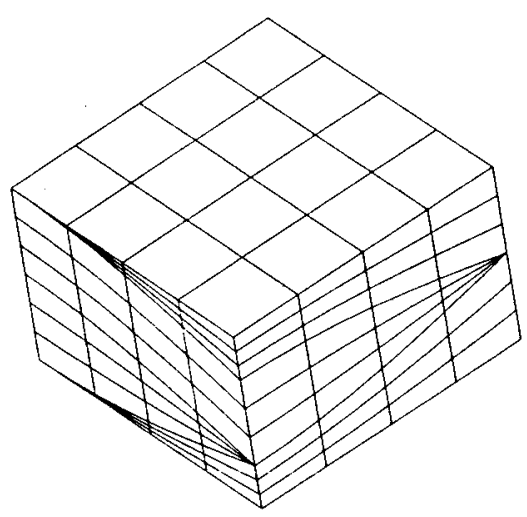

Mesh 4 :

192 elements and 1049 nodes

Figure 2 Finite element meshes used to determine the effect of mesh refinement on failure prediction. 


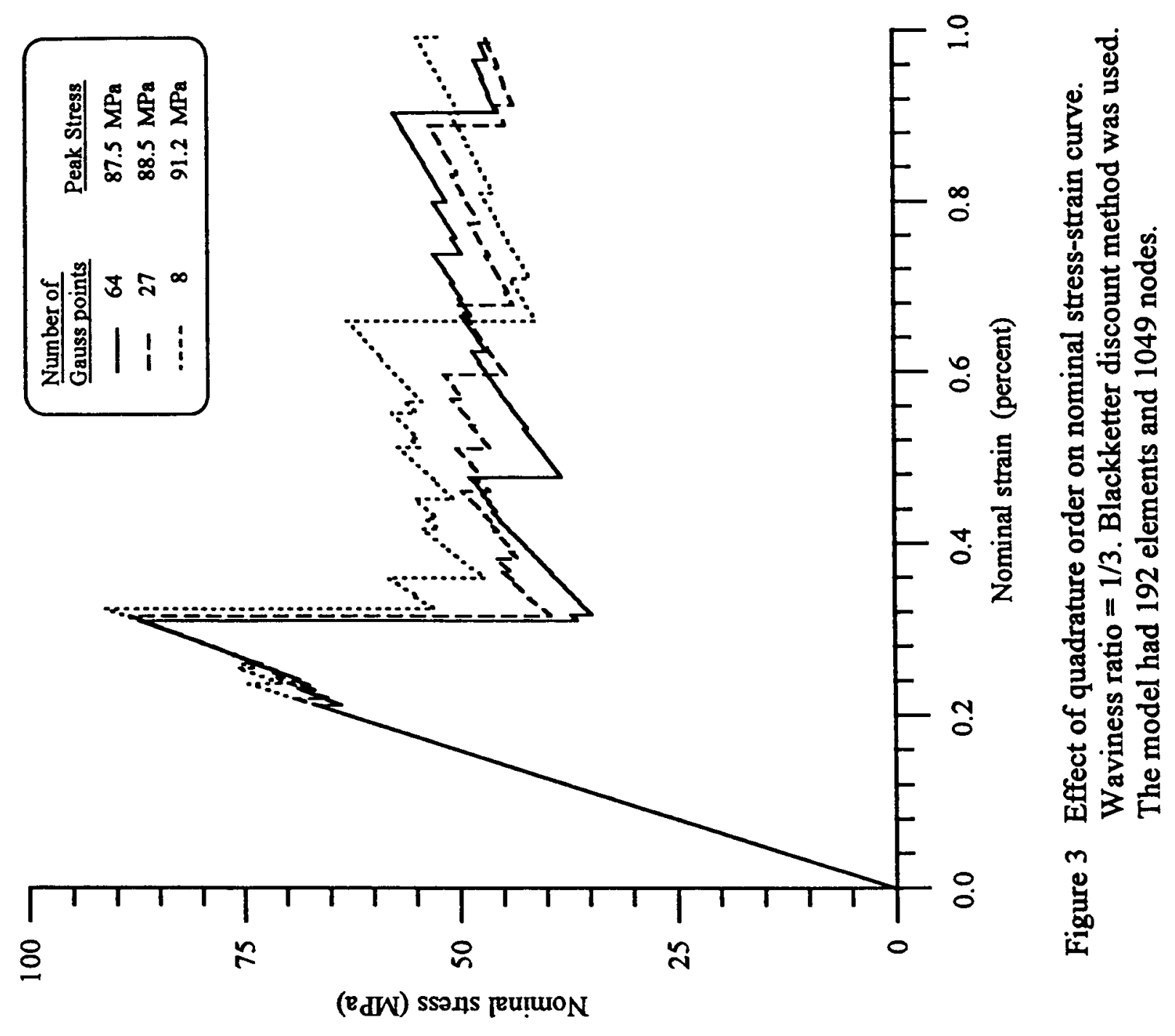




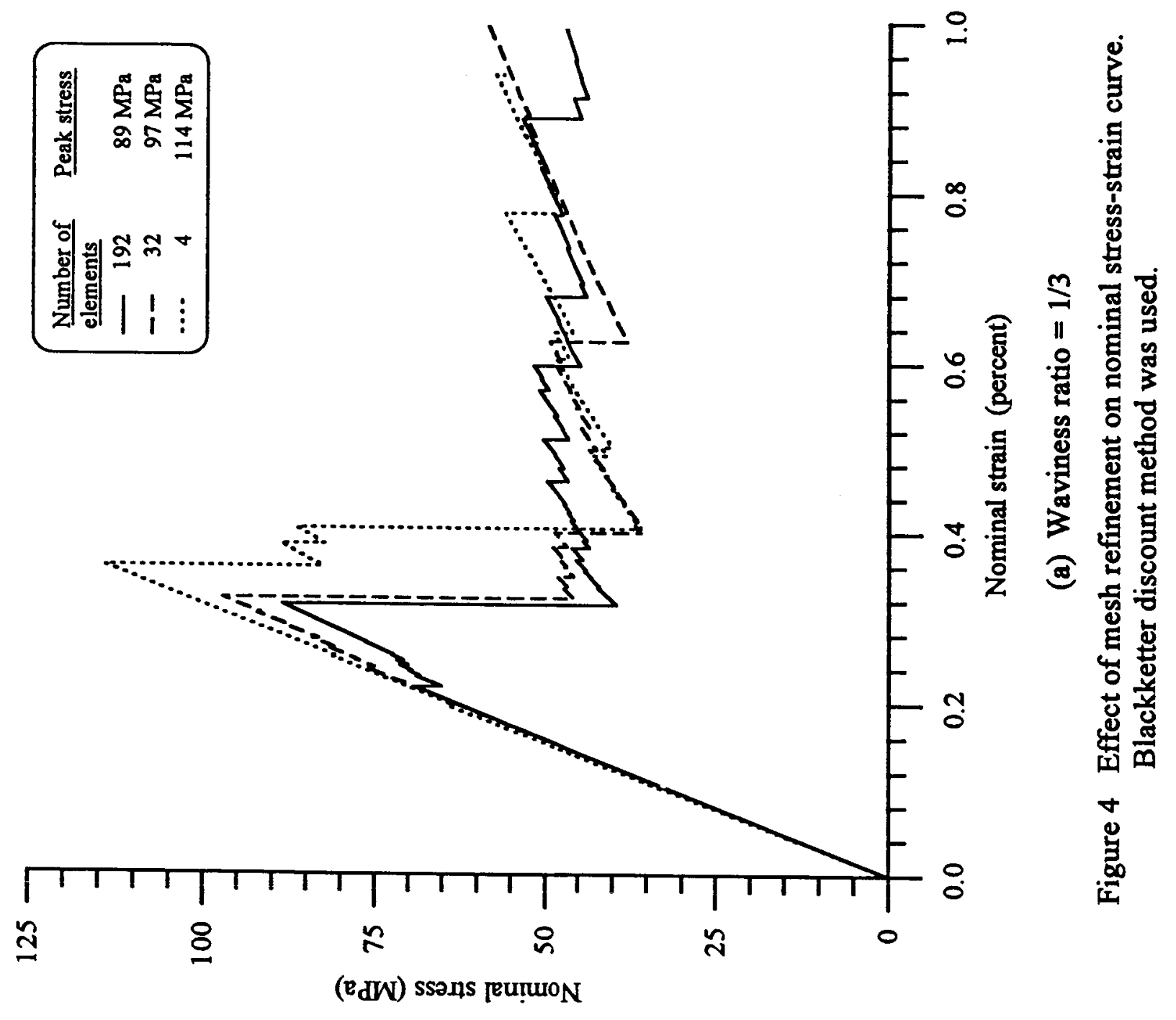




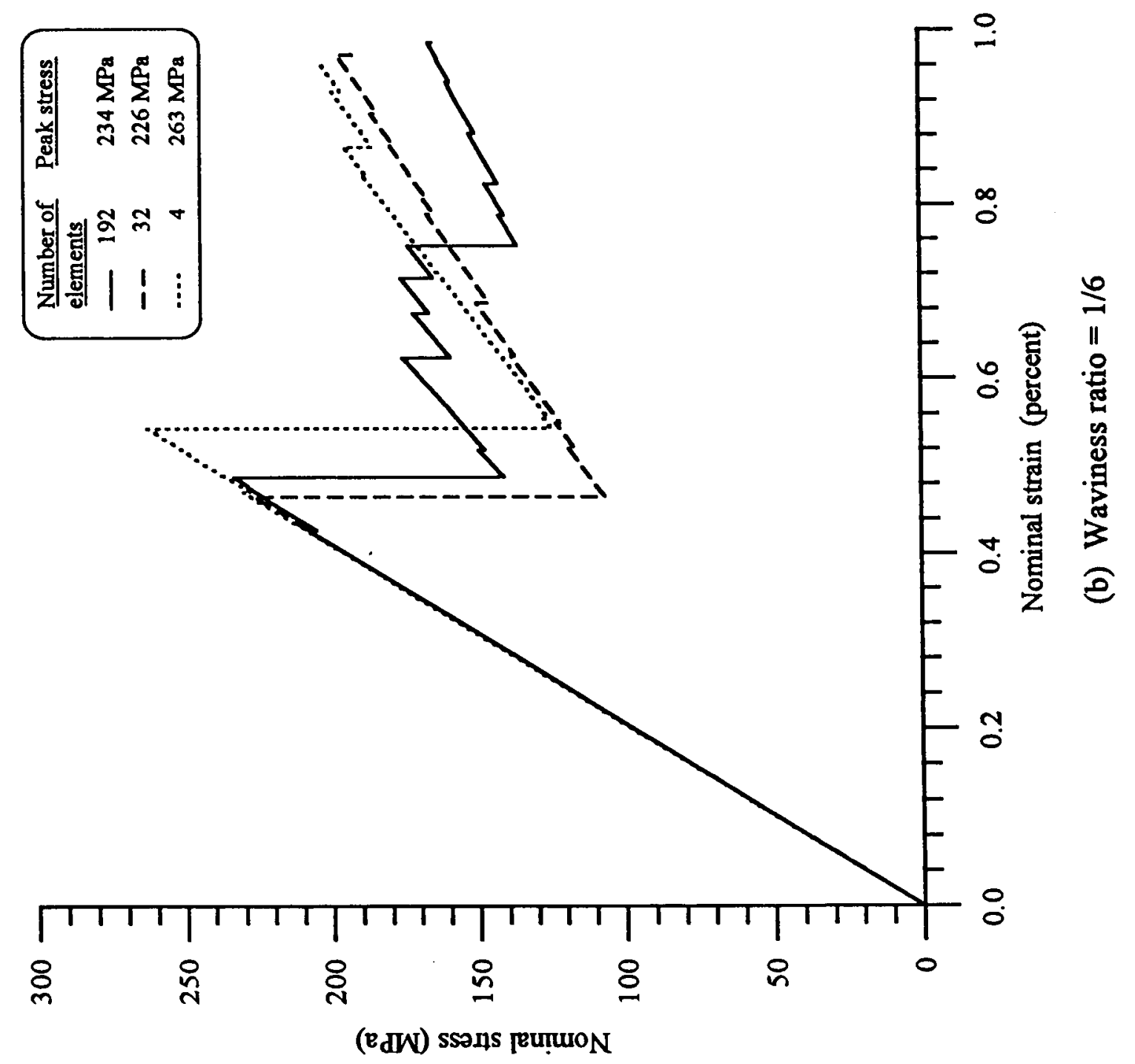

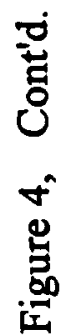




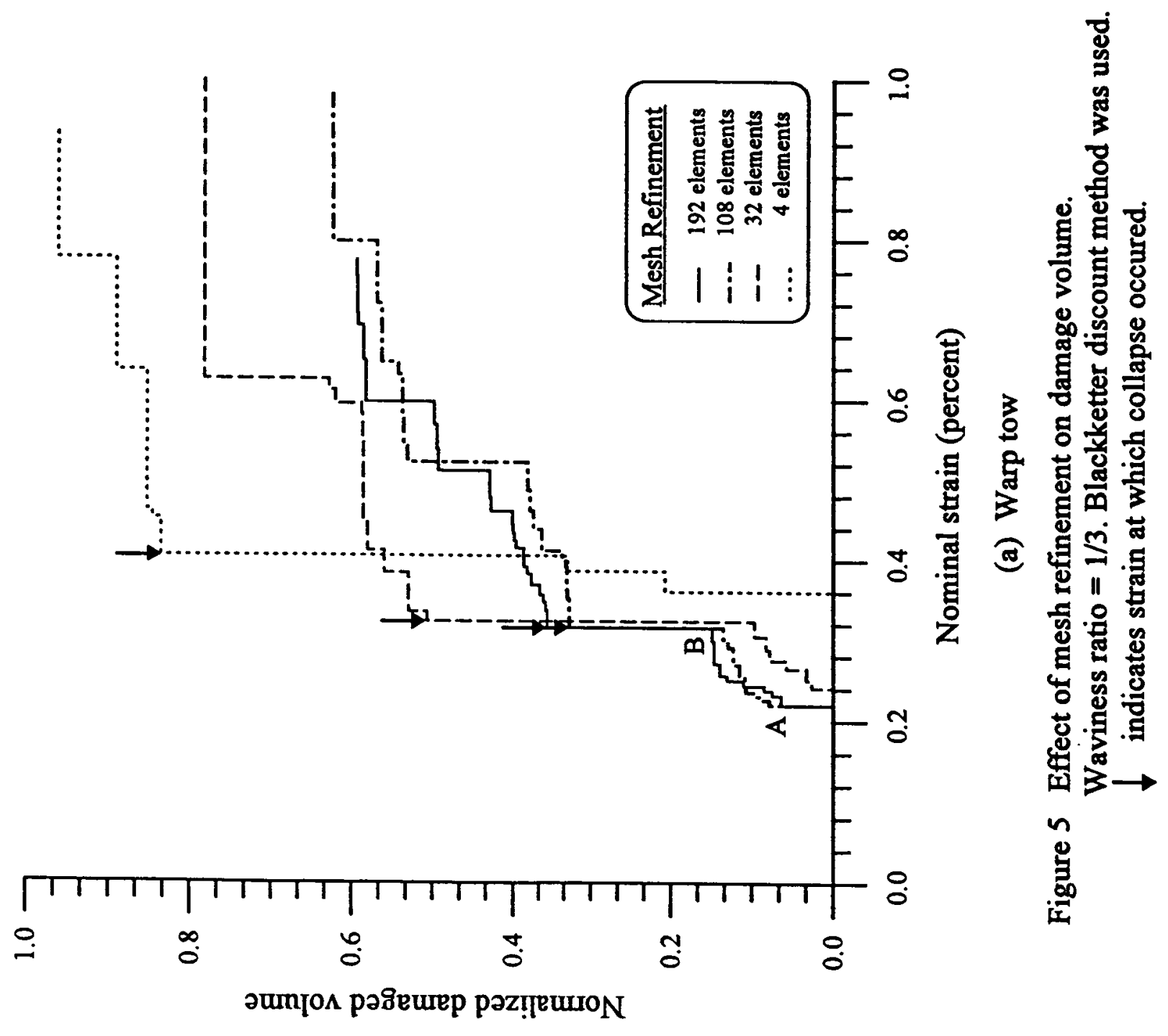




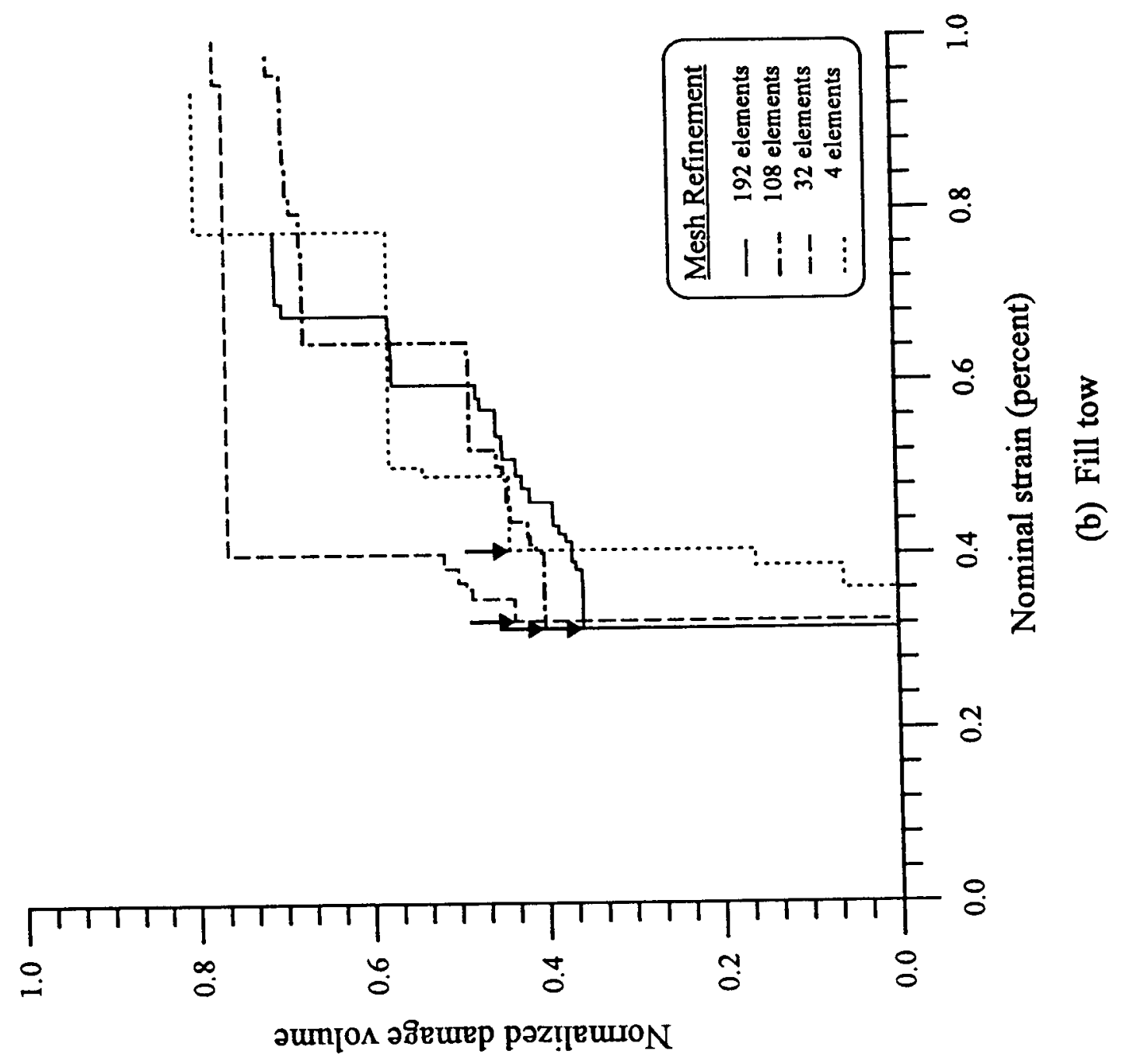

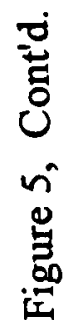




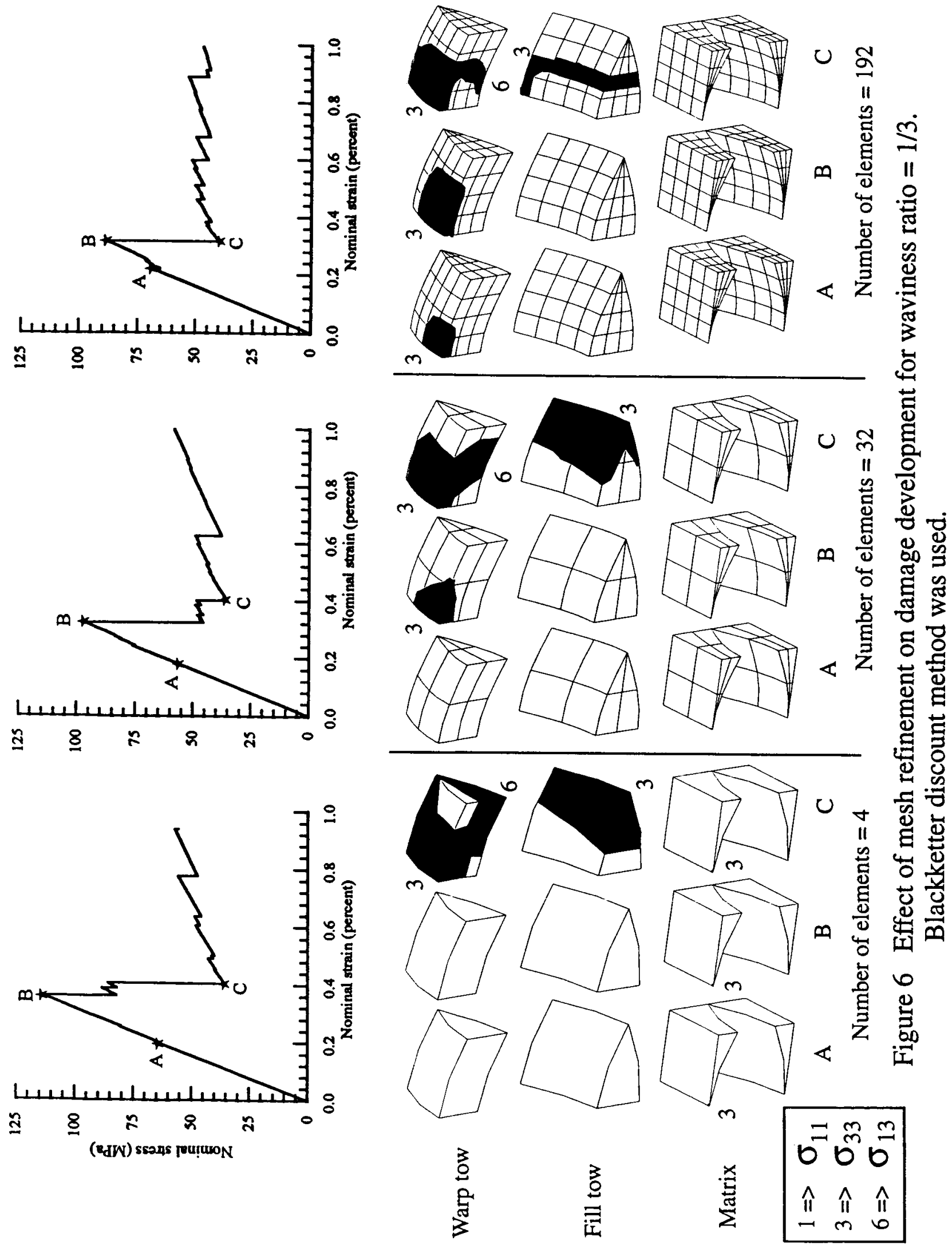




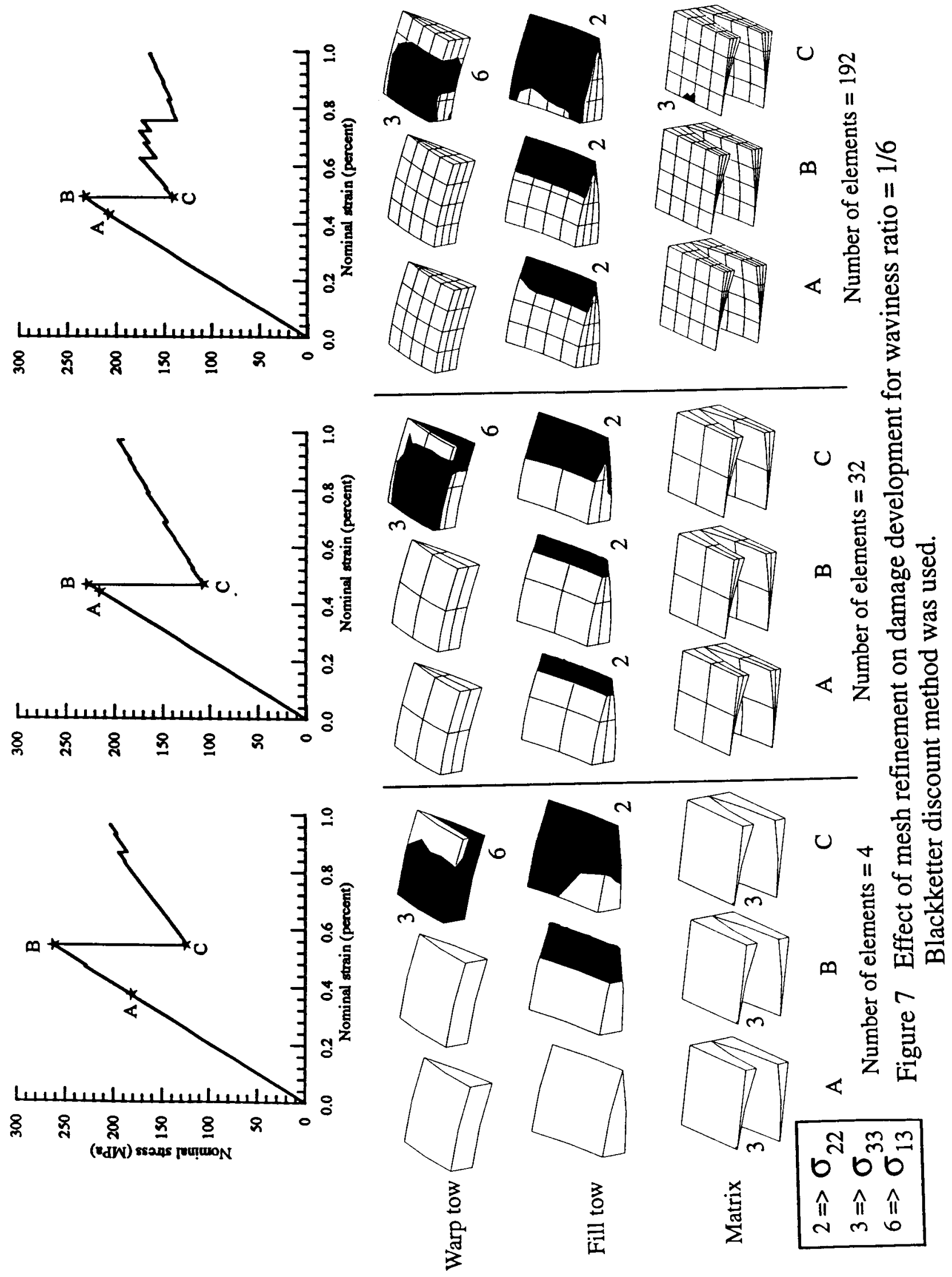




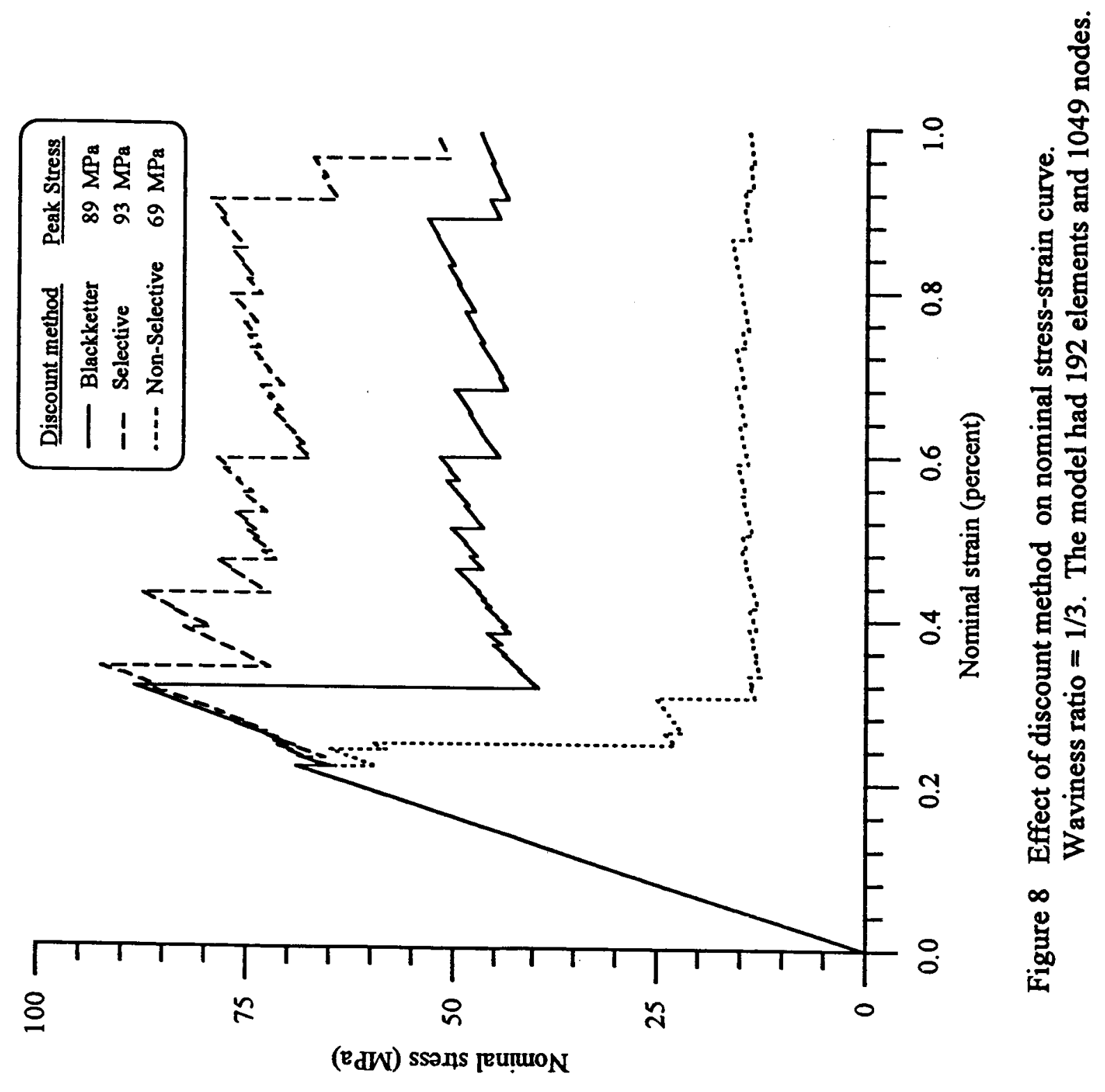






